\title{
Nanobiosensors: Concepts and Variations
}

\section{Parth Malik, ${ }^{1}$ Varun Katyal, ${ }^{2}$ Vibhuti Malik, ${ }^{3}$ Archana Asatkar, ${ }^{4}$ Gajendra Inwati, ${ }^{1}$ and Tapan K. Mukherjee ${ }^{5}$}

${ }^{1}$ Center for Nano Sciences, Central University of Gujarat, Gandhinagar, India

${ }^{2}$ Department of Chemical Technology, Delhi Technological University, Delhi, India

${ }^{3}$ Department of Phramaceutics, Hindu College of Pharmacy, Sonepat, Haryana, India

${ }^{4}$ School of Chemical Sciences, Central University of Gujarat, Gandhinagar, India

${ }^{5}$ Indian Institute of Science Education and Research, Mohali, Chandigarh, India

Correspondence should be addressed to Tapan K. Mukherjee; tapanmu@yahoo.com

Received 11 June 2013; Accepted 21 August 2013

Academic Editors: K. C. Hwang and D. Pissuwan

Copyright (C) 2013 Parth Malik et al. This is an open access article distributed under the Creative Commons Attribution License, which permits unrestricted use, distribution, and reproduction in any medium, provided the original work is properly cited.

Biosensing has been one of the hottest topic attracting scientific minds since long back. It is so as biological entities are very complex and are directly associated with the existence of a healthy environment. The design of biosensors also has witnessed significant changes in the recent past. Biosensors for applications as diverse as food quality estimation, environmental monitoring, and diagnosis of clinical and metabolic complications have come to the fore. Nanotechnology has bestowed some highly exciting ingredients for the improvement of sensing phenomenon. The use of diverse nanomaterials ranging from nanoparticles, nanotubes, nanorods, and nanowires has enabled faster detection and its reproducibility in a much better way. The unique properties of nanomaterials such as high electrical conductivity, better shock bearing ability, and the sensitive responses such as piezoelectric and versatile color based detection mechanisms are only the results of congregation of nanomaterial properties. This paper highlights the different types of biosensors based on different types of nanomaterials and their developmental and implicational aspects.

\section{Introduction}

Sensing the biological responses has assumed great significance in the current scenario of ever dynamic environmental developments and corresponding altered homeostatic happenings occurring at both in vivo as well as ex vivo levels. The analysis of behavior of the ever changing materials has assumed great significance in areas like pharmaceutical diagnosis, screening food quality, and environmental applications. In this reference, the development of efficient biosensors which can analyze the minutest details of the biological interactions even at a very small scale and with extreme precision and maximum ever possible sensitivities deserves urgent attention [1]. A key component of the biosensing is the transduction mechanisms which are responsible for converting the responses of bioanalyte interactions in an identifiable and reproducible manner using the conversion of specific biochemical reaction energy into an electrical form through the use of transduction mechanisms. Nanomaterials can be wonderful incumbents in this dimension as they have high surface area to volume ratios which allow the surface to be used in a better and far more diversely functional manner. Moreover, their electromechanical properties are the wonderful assets for the biosensor technology. Nanostructural wonders provided by nanotechnology have revolutionized the happenings in the domain of molecular biology which have provided an opportunity for manipulation of atoms and molecules and monitored the biological phenomenon at the physiological level with far greater precision. The terminology nanobiosensors a misnomer in the sense that it has the word nano prefixed to it. To get to the real technology, one must soundly gather the idea of what a biosensor is. As nanoscience is interdisciplinary in nature so putting the word nano as prefix often implies the use or manipulation at a scale equivalent to one-billionth of a meter. 


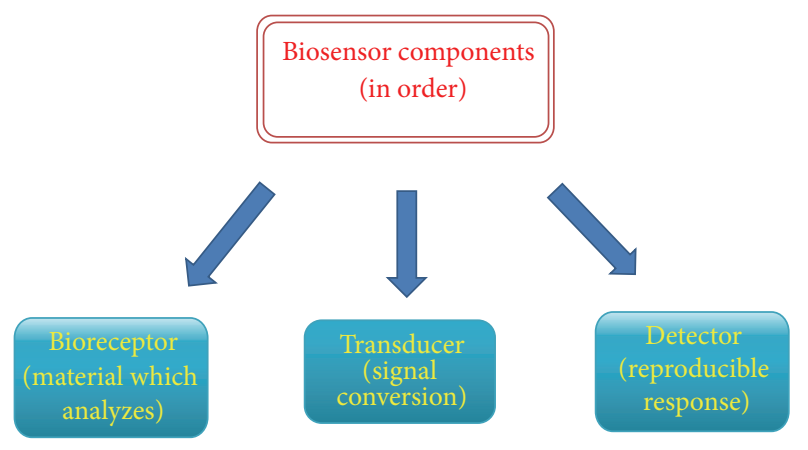

FIGURE 1: Depiction of the block diagram of a biosensor.

\section{Definition and Conceptual Idea}

A biosensor can be defined as a sensing device or a measurement system designed specifically for estimation of a material by using the biological interactions and then assessing these interactions into a readable form with the help of a transduction and electromechanical interpretation. Figure 1 gives us information about the three main components of a biosensor. In terms of the conceptual and fundamental mode of operation, these components are, namely, bioreceptor, transducer, and the detector. The main function or purpose of a biosensor is to sense a biologically specific material. Often, these materials are antibodies, proteins, enzymes, immunological molecules, and so on.

It is done by using another biologically sensitive material that takes part in the making of bioreceptor. So, a bioreceptor is that component of a biosensor which serves as a template for the material to be detected. There can be several materials which can be used as bioreceptors. For instance, an antibody is screened using antigen and vice versa; a protein is screened using its corresponding selective substrate and so on. The second component is the transducer system. The main function of this device is to convert the interaction of bioanalyte and its corresponding bioreceptor into an electrical form. The name itself defines the word as trans means change and ducer means energy. So, transducer basically converts one form of energy into another. The first form is biochemical in nature as it is generated by the specific interaction between the bioanalyte and bioreceptor while the second form is usually electrical in nature. This conversion of biochemical response into electrical signal is achieved through transducer. The third component is the detector system. This receives the electrical signal from the transducer component and amplifies it suitably so that the corresponding response can be read and studied properly. In addition to these components, a very essential requirement of the nanobiosensors is the availability of immobilization schemes which can be used to immobilize the bioreceptor so as to make its reaction with bioanalyte much more feasible and efficient. Immobilization makes the overall process of biological sensing cheaper, and the performance of the systems based on this technology is also affected by changes in temperature, $\mathrm{pH}$, interference by contaminants, and other physicochemical variations [2].

\section{Nanobiosensors: The Merging of Nanotechnology with Biosensors}

The understanding of biosensing concept lays the foundation for studying and developing the nanobiosensors. Nanobiosensors are basically the sensors which are made up of nanomaterials and interestingly these are not the specialized sensors which can detect the nanoscale events and happenings. The question that sustains interest from the above description is that why nanomaterials are intended to be used in making biosensors or whether they are going to drive in any significant difference in the overall technology. Nanomaterials are a unique gift of nanotechnology to the mankind; these are the materials which have one of their dimensions between 1 and 100 nanometers. The size constraints of these materials makes them very special as they have most of their constituent atoms located at or near their surface and have all vital physicochemical properties highly different from the same materials at the bulk scale. They can play very efficient roles in the sensing mechanism of the biosensor technology. Integrated devices of the nanomaterials with electrical systems give rise to nanoelectromechanical systems (NEMS), which are very active in their electrical transduction mechanisms. Several nanomaterials have been explored on the basis of their electronic and mechanical properties for their use in improved biological signaling and transduction mechanisms. Some of such materials that are widely employed include nanotubes, nanowires, nanorods, nanoparticles, and thin films made up of nanocrystalline matter [3]. Amongst these, the use of nanoparticles is best studied and analyzed till date. Nanobiosensors have served as very potent developmental inroads in the biosensor technology, which has been possible only due to the wonders of nanotechnological implications of the matter. A wide variety of biosensing devices that employ nanoparticles or nanostructures have been investigated in a number of studies throughout the world. These can be as diverse as using amperometric devices for enzymatic detection of glucose to using quantum dots as fluorescence agents for the detection of binding and even using bioconjugated nanomaterials for specific biomolecular detection. These include colloidal nanoparticles which can be used to conjugate with antibodies for immunosensing and immunolabelling applications. These materials can also be used to enhance the electron microscopic detections. Further, metal based nanoparticles are very excellent materials for electronic and optical applications and can be efficiently used for detection of nucleic acid sequences through the exploitation of their optoelectronic properties.

Table 1 shows the main types of nanomaterials being employed for further improvising upon the sensing mechanisms that are conventionally being employed in the biosensor technology. It highlights the potential advantages of several nanomaterials employed and some evidence witnessed their use so far (encoded by corresponding references). The details of different biosensors developed by the use of different materials at the nanoscale are mentioned further in the text. Even, magnetic nanoparticles made up of iron and its oxides have been used for specific and efficient detection of magnetism based events and interactions like those of 
TABLE 1: Depiction the overview of nanomaterials used for improving biosensor technology.

\begin{tabular}{|c|c|c|c|}
\hline Sr. no. & $\begin{array}{c}\text { Nanomaterial } \\
\text { used }\end{array}$ & Key benefits & References \\
\hline (1) & $\begin{array}{c}\text { Carbon } \\
\text { nanotubes }\end{array}$ & $\begin{array}{l}\text { Improved enzyme loading, } \\
\text { higher aspect ratios, ability } \\
\text { to be functionalized, and } \\
\text { better electrical } \\
\text { communication }\end{array}$ & {$[4-6]$} \\
\hline (2) & nanoparticles & $\begin{array}{l}\text { Aid in immobilization, } \\
\text { enable better loading of } \\
\text { bioanalyte, and also possess } \\
\text { good catalytic properties }\end{array}$ & {$[7-10]$} \\
\hline (3) & $\begin{array}{l}\text { Quantum } \\
\text { dots }\end{array}$ & $\begin{array}{l}\text { Excellent fluorescence, } \\
\text { quantum confinement of } \\
\text { charge carriers, and size } \\
\text { tunable band energy }\end{array}$ & [11-13] \\
\hline (4) & Nanowires & $\begin{array}{l}\text { Highly versatile, good } \\
\text { electrical and sensing } \\
\text { properties for bio- and } \\
\text { chemical sensing; charge } \\
\text { conduction is better }\end{array}$ & [14-16] \\
\hline (5) & Nanorods & $\begin{array}{l}\text { Good plasmonic materials } \\
\text { which can couple sensing } \\
\text { phenomenon well and size } \\
\text { tunable energy regulation, } \\
\text { can be coupled with } \\
\text { MEMS, and induce specific } \\
\text { field responses }\end{array}$ & [17-19] \\
\hline
\end{tabular}

magnetic resonance imaging (MRI). These particles can be coupled with fluorescent molecules or can be made to deliver specific responses by coupling with externally applied magnetic fields. Similarly zinc and zinc oxide based nanostructures have been extensively used for sensing of biochemical phenomenon in a much more precise and sensitive manner. These have been used in optimized detection of cholesterol and many other metabolic intermediates. Continuing along the same direction, carbon nanotubes have also been used to optimize the biosensing events with reference to their ability to allow for rapid detection and much better interactions between the analyte and the bioreceptor molecule. Carbon nanotube based biosensors have been actively in use for the detection of glucose [20] and insulin [21]. The text ahead mentions the advantages and outcomes of the use of different nanomaterials and their inherent benefits and the critical parameters in which they can have significant impacts and yield significantly better results.

\section{Selection and Optimization of Nanomaterials for Sensor Technology}

There is a multitude, factors which govern or decide the use of a particular kind of nanomaterials for biosensing applications. These factors are the chief ingredients of their physical and chemical properties along with their energy sensitive and selective responses. Table 1 has already mentioned the main important nanomaterials employed for biosensing applications. Before exactly implementing or adding a nanomaterial for the sensing applications, we first focus on their desired manufacturing which is a part of experimental design known as "Nanofabrication." The technique of nanofabrication targets two vital operations, namely, the manufacturing and design of nanoscale adhesive surfaces via the technology of integrated circuits and the engineering of nanomaterial surfaces through the process of micromachining. This technique, thus developed for biosensing, uses the variations of four basic processes, namely, photolithography, thin film etching/growth, surface etching strategies, and chemical bonding parameters.

Nanoscale electrodes which have come into picture as a result of lithography technique have enhanced the biosensing accuracy by providing much better and greater surface areas that in turn enable the immobilization to be achieved with greater precision [22]. Glucose biosensors, making use of enzyme glucose oxidase, have been developed using these innovations. The strategies involving the use of active nanoparticles of platinum over the sheets of carbon nanotubes have significantly enhanced the immobilization of enzyme systems required for the detection of the analyte materials. These systems have significantly much wider applications to biosensing technology, enabling the detection of glucose from several sources other than blood. In a similar manner, couples of immunosensors have also been developed which involve coating of thin films over the sensing surface that enables faster and better detection of the corresponding analytes [23].

Highly sensitive electrical and electromechanical properties are incorporated into several materials by engineering them with nanoelectromechanical systems (NEMS), which have enabled the generation of complex electrical, mechanical, fluidic, thermal, optical,and magnetic properties of the materials with sizes down to the nanometer level. NEMS technology has thus provided many materials with novel properties due to their nanoscale functionalization. NEMS and MEMS devices have enabled better and better sophisticated performance of the mechanical materials as the mechanical properties of a material are a critical function of its size. In addition, these devices have been coupled with biological systems and molecules to improve their bio adhesion characteristics and the response to a wide range of stimuli. With the implementation of NEMS and MEMS, surface forces like friction, adhesion, cohesive forces, and viscous drag forces can be controlled in a very precise manner that enables the best modeling of the biochemical interactions taking part in the biosensing technology [24].

Another important factor considered while using nanomaterials for sensing application is the monitoring and optimization of their optical properties. The phenomena like surface plasmon resonance are very interesting and in particular expected from nanoparticles so as to maximize the sharp and precise scale optical response of the sensing materials with the incident light. The surface plasmon resonance effect is concerned with the excitation of particle surface with the ionic species and charged particles which create ions and result in excitation of the fluidic state of charged particles. 
This property is highly suitable in case of nanoparticles due to their unique optical properties which give them photonic character and excellent ability to be used as fluorophores. This phenomenon makes use of total internal reflection which takes place for angle of incidence reaching beyond a critical value. Here, the reflection of light through a thin film of metallic nanoparticles coated over a surface is optimized by the corresponding adjustment of the critical angle of reflection. In case of nanomaterials, this phenomenon is highly logical and is especially named as localized surface plasmon resonance [25]. Surface plasmon resonance effect is also dependent upon the refractive index of a medium and it is the most fundamental property governing the flow of light through a medium. Due to the phenomenon of surface plasmon resonance, a nanobiosensor is better equipped to detect the minutest interacting phenomenon, which enables a far greater and much reliable degree of estimation of biological interactions through a nanobiosensor in comparison with a biosensor [26-28].

In this way, nanomaterials, irrespective of their nature, need to be optimized for their performance and effect as per the desired goal before being actually implemented for the biosensing purpose. Nanostructured semiconductor crystals can be efficiently used to improve the detection of neurological responses via coupling through the sensing molecule of biological nature. These can be coupled with peptide assembly of a range of nanomaterials so that efficient interaction can be generated by means of self-assembly and this also saves a lot of time that is being involved in the currently available technologies and methods. Moreover, these can rapidly detect the biological stimulus such as that of a DNA segment or a characteristic nucleotide sequence pertaining to proteins or even RNA [29]. Moreover a key strategy into shaping-up of nanomaterials for desired applications involves the tuning and engineering of their surface by sophisticated inroads collectively termed as micromachining procedures. Factors like aspect ratios, functionalization with other materials and compatibility issues with respect to the material being analyzed for are highly critical for the use of nanomaterials in biosensing applications.

\section{Nanobiosensors: Variations and Types}

The classification of nanobiosensors is a very diverse area. This is so as it is based on the nature of nanomaterials incorporated in the biosensing operation. Moreover the classification here is not as simple as it is in the case of biosensors. In case of biosensors, we classify the sensors on two criteria, namely, the type of material to be analyzed and the other one is on the basis of signal transduction mechanism employed. For instance, if we are screening any antigen or enzyme through the biosensors, we name them as antigen biosensors or enzyme biosensors as per the convention of naming a biosensor through the nature of the analyte. Similarly, if we arrive at the classification of biosensors with respect to their sensing mechanism, the main types are electrochemical, calorimetric, optical, and acoustic. Each of these classes is based on the transduction mechanism involved and includes a series of overlapping sensor categories under it. For instance, amperometric and potentiometric biosensors come under the category of electrochemical sensors; likewise optical biosensors carry optical fiber based sensors and surface plasmon resonance based sensors under them [30].

Considering the classification of nanobiosensors, we observe that the criteria for classification are the nature of nanomaterials being involved for improving the sensing mechanism. For instance, nanoparticle based biosensors include all the sensors which employ metallic nanoparticles as the enhancers of the sensing biochemical signals. Similarly, nanobiosensors are called nanotube based sensors if they involve carbon nanotubes as enhancers of the reaction specificity and efficiency while biosensors using nanowires as charge transport and carriers are termed as nanowire biosensors. Likewise there are quantum dots based sensors which employ quantum dots as the contrast agents for improving optical responses. The text ahead enlists some of the major classes of nanobiosensors developed till date and those which are in practical use.

\subsection{Nanoparticle Based Sensors}

5.1.1. Acoustic Wave Biosensors. Acoustic wave biosensors have been developed to amplify the sensing responses so as to improve the overall preciseness of the limits of biodetection. There can be so many stimulus based effects in these kinds of sensors. The mass based variant of these sensors involves the conjugation of antibody modified sol particles which bind themselves on the electrode surface that has been complexed with the particles of analyte conjugated in a manner that antibody molecules are immobilized over the electrode surface. The large mass of bound sol particles of the antibody results in a change in the vibrational frequency of the quartz based sensing platform, and this change acts as the basis of detection. In general, the preferred diameter of the sol based antibody particles is between 5 and $100 \mathrm{~nm}$. Particles of gold, platinum, cadmium sulphide, and titanium dioxide are generally preferred $[31,32]$.

5.1.2. Magnetic Biosensors. Magnetic biosensors utilize the specially designed magnetic nanoparticles. These are mostly ferrite based materials, either used individually or in combined form. These types of sensors are very useful with reference to the biomedical applications. The magnetic materials enable a great deal of diversity for several analytical applications. This is so because the magnetic compounds involved in screening constituted of iron coupled with other transition metals, which have different properties. With the incorporation of magnetic nanoparticles, the conventionally used biodetection devices have further become more sensitive and powerful. Alloys of transition metals with iron and other materials having unpaired electrons in their d-orbitals have been highly versatile in their magnetic behaviors. A very popular kind of materials that have come to the fore involving these employs magnetic bioassay techniques that are used for specific isolation of magnetically labeled targets 
with the help of a magnetometer [33]. Special devices such as superconducting quantum interference devices (SQUID) have been used for rapid detection of biological targets using the super paramagnetic nature of magnetic nanoparticles. These devices are used to screen the specific antigens from the mixtures by using antibodies bound to magnetic nanoparticles [34]. These make use of super paramagnetic effect of magnetic materials which is particularly observed in the nanoscale particles.

5.1.3. Electrochemical Biosensors. These sensors basically work to facilitate or analyze the biochemical reactions with the help of improved electrical means. These devices are mostly based on metallic nanoparticles. The chemical reactions between the biomolecules can be easily and efficiently carried out with the help of metallic nanoparticles, which significantly help in achieving immobilization of one of the reactants. This ability makes these reactions very specific and eliminates any possibility of getting undesirable side products. In this reference, colloidal gold based nanoparticles have been used to enhance the immobilization of DNA on gold electrodes which has significantly increased the efficiency of an overall biosensor by further lowering the detection limit [35]. Biosensors have been designed using enzyme conjugated gold nanoparticles for the identification of glucose, xanthine, and hydrogen peroxide [36-38]. In a significant study by $\mathrm{Xu}$ et al., the analysis of electrochemistry of enzyme systems comprising of horse reddish peroxidase, immobilized on gold electrodes loaded with nanoparticles of carbon, has been put forward [38]. The study predicted a faster amperometric response and faster and much better electrocatalytic reduction ability for horse reddish peroxidase. As a result, the biosensor developed showed improved sensitivity and much lower detection limit as compared to the one without using nanoparticles.

In a similar trend, nanosized semiconductor crystals can be used to improve the efficiency of photochemical reactions and can be tagged to biological entities like those of enzymes and precursors to design novel photo electrochemical systems. In this dimension, Curri et al. (2002) have used immobilized nanocrystalline CdS using self-assembly approach so as to prepare an enzymatic detection system based on immobilized formaldehyde dehydrogenase onto the gold electrodes in order to carry out the catalytic oxidation of formaldehyde [39]. In several other studies, metal based nanoparticles have been used for coupling themselves with biological probes and then carry out useful detection of the specific molecules from a mixture. Bioassays based on biotinstreptavidin specificity have been designed in this regard [40].

5.2. Nanotube Based Sensors. Carbon nanotubes are one of the most popular nanomaterials known right now in the world of material science and optoelectronic applications. Since their discovery in 1990's, they have attracted interest worldwide because of their extraordinary properties, the most vital of which are the electronic conductivity, flexible physical geometric features, and the ever dynamic physicomechanical properties ranging from high aspect ratios to very good functionalization abilities along with having high mechanical strength and folding abilities. Because of these attributes, both single walled nanotubes as well as multiwalled nanotubes have been used in designing biosensors for better and better performances [41, 42].

The most popular sensing advances that have come to the fore are the developments in the design of glucose biosensors that involve the use of nanotubes as immobilizing surfaces for enzyme glucose oxidaze; this enzyme is used for estimation of glucose from the several body fluids. In convention, the sensors using enzymes predicted the presence of glucose from major body tissues but the use of nanotubes as assemblies for immobilization has led to the estimation of glucose from even scarce body fluids such as tears and even saliva. In one such arrangement, single walled nanotubes have been wonderfully employed for enzymatic detection of glucose, and this innovation has also yielded significant increase in the enzyme activity [43]. The enhanced performance of the biosensor was analyzed and found so, largely due to the high enzyme loading and better electrical conductivities of the nanotubes. Not only with their structural flexibilities, carbon nanotubes have also been used for enhancing the electrical detection of the sensing phenomenon, owing to their better and smoother electron transfer flow characteristics. The significant improvements in the catalytic biosensors have been widely exploited in several studies, and in one such study, this innovation resulted in better oxidoreductase performance in both glucose oxidase and flavin adenine dinucleotide precursors binding to their substrates more efficiently and in a much more controllable manner [44]. Likewise, chemoelectroluminiscence effect has been improved by coupling CNTs to the sensing molecules of a sensor through better conductance of charge carriers and controlling their required flow characteristics. In a significant and comprehensive review, biosensors based on carbon nanotubes have been extensively summarized for key breakthroughs and advantages that they get bestowed with, by the incorporation of nanostructured arrays of carbon nanotubes and the related structure sensitive assemblies. This review highlights the functionalization potential of carbon nanotubes and their rapid friendliness for being coupled with biomolecules like DNA, proteins, oligonucleotide probes, and their corresponding benefits in an excellent manner [45].

5.3. Nanowire Based Sensors. Nanowires are cylindrical arrangements just like those of carbon nanotubes, having lengths in the order of few micrometers to centimeters and diameters within the nanorange. Nanowires are the onedimensional nanostructures with very good electron transport properties. Significantly, the motion of charge carriers in the nanowires is vigorously improved and different from those in bulk materials. Sensors based on nanowires are very less in number, but literature reports some exciting examples where use of nanowires has significantly improved the performance and detection of biological materials. In one such study, Cui and Lieber group have reported the performance of biosensors based on silicon nanowires doped with boron and used them for the detection of biological 
and chemical species [46]. Semiconductor nanowires have been exploited in a great detail and have also been used for coupling a number of biomolecules for identifying their specifically linked substrates. In a study, silicon nanowires coated with biotin have been used for the detection and isolation of streptavidin molecules from a mixture. The small size and capability of these nanowires make them ideal candidates to be used for biodetection of pathogens and many other real time analysis of a wide range of biological and chemical species, thus vastly improvising the current accuracies of presently used in vivo diagnostic procedures. As these sensing materials work on very precisely defined dimensions, they can also be used to accomplish in vivo applications and operate in the smallest environments within the living cells. In one such study, Wang et al. have used optical fibers with diameters in the nanosize and coated with antibodies to detect the presence of toxicants within the single cells [47]. In another very closely related study, Cullum et al. have reported the synthesis of $\mathrm{ZnO}$ nanowires, coated them over the gold electrodes, and then used them for detection of hydrazine using amperometric responses [48]. They have proposed a high sensitivity, low detection limit, and far lower response times response times than those reported in the conventionally used reported in the conventionally used sensor systems. Nanowires are very versatile in their performance and are significantly better than nanotubes in two major ways. First, they allow a range of modifications in their design by control of operational parameters during their synthesis. Secondly, they possess a lot much more scope for the development of functionalized assemblies by virtue of the existence of compatible materials on their surfaces. Despite very well-known synthesis procedure for nanowire synthesis, their use in the development of sensing devices has met several challenges [49]. It has been reported by several related studies that it is difficult to incorporate nanowires into the sensing systems for the overall improvement in their electrical conductivities to be realized. In a very advanced study, Lieber group have rigorously worked on semiconductor nanowires and synthesized them by using combinations of previously known methods. They have developed a complex one dimensional architecture comprising of at least 200 independent electrical nanowire assemblies and have used them to perform a low level detection of serum-bone cancer antigens [46]. For the best analysis of rationale behind using nanowires or knowing about their qualities that can improve sensing mechanism, Yang et al. provided a valuable insight by rigorously talking about nanowires and nanobelts as well as their structural aspects and features through which they can be used in sensing applications [50]. In a couple of related advancements, both Cui et al. and Huang et al. have explored the salient attributes of nanowires and explained their utility in better conduction and detection of biological stimulus [51, 52]. Huang et al. have particularly focused on surface plasmon resonance ability of nanowires, through which these can be incorporated into the sensing probes and significantly improve the sensitivity of sensing event. To make the process little more simplified, Stern et al. have developed nanowires through the complementary metal oxide semiconductor (CMOS) approach. This approach has proved to be very easy in terms of controlling and regulating the synthesis procedures for nanowires and has been used for analysis of serum fluids so as to enable the isolation of several pathogens and proteins in crude form [53].

In this way, nanomaterials have proved to be highly prosperous for brightening the sensing technology and have improved the diagnostic and detection procedures by leaps and bounds. The faster and quicker diagnosis enabled by still faster analysis and evaluation protocols through the nanomaterials has just revolutionized the biosensing mechanism. There are many other nanomaterials except those mentioned above that have been capitalized upon and made use of in biosensing applications. Nanodots resembling the morphology of quantum dots, nanosheets, and many other structures of altered geometries such as nanocombs, nanobelts and nanoribbons have been used for improving the conventional procedures of sensing. The coupling of piezoelectric and cantilever systems has further added a new charm to this technology. Nanomaterials like quantum dots have been added as labels coupled with sensitive dyes, and they have yielded thermochromic, photochromic, and electro chromic materials which can show highly sensitive detections that can be monitored easily. They have significantly helped in improving electron transport mechanisms and also in the development of much more efficient actuating mechanisms to impose a particular state of observation on a system. The text ahead mentions some impact provoking applications of nanobiosensors in the different walks of life.

\section{Applications of Nanobiosensors}

The definition and description of the concept of operation of nanobiosensors do not leave any room for their applications as they are highly versatile and multifunctional, so many and perhaps endless. From the estimation and diagnosis in the health related in vivo aspects, biosensors can also be used for environmental monitoring of pollutants, toxicants, and physical aspects like humidity, heavy metal toxicity, and even presence of carcinogens.

(1) Biomedical and Diagnostic Applications. Biosensors have been used for biological detection of serum antigens and carcinogens, and causative agents of so many metabolic disorders since time immemorial. The routine applications in diagnosis are best described by the use of biosensors in the detection of disorders like diabetes, cancer, allergic responses, and so many other disorders on the basis of serum analysis. To talk about most of the studied and effectual applications of nanobiosensors from clinical point of view, there are numerous clinical applications that are principally being enabled using biosensors in routine. These applications include the detection of glucose in diabetic patients [54, 55], detection of urinary tract bacterial infections $[47,56]$, detection of HIV-AIDS [57, 58], and the diagnosis of cancer [59-61]. Indeed, all of these are highly critical health problems affecting the mankind at present throughout the world. Prior to the use of biosensors, the detection and diagnosis of these diseases were very difficult, time consuming and costly. The advent of biosensors has really improved the diagnosis of all 
these diseases and related malfunctions. With the addition of nanoscale interventions, this diagnosis has further been benefitted and made more precise. The incorporation of nanomaterials has enabled the detecting enzyme systems to be immobilized, and this has allowed the recycling and reuse of costly enzymes. Besides, they have improved sensitivity and accuracies that make them hot candidates for being tapped upon. The implementation of nanoscale innovations like NEMS and MEMS has enabled several advantages to the overall testing procedures. Extremely sensitive inroads like those of lab-on-a-chip based assays have been developed using smart sensing nanoscale materials only. Biochips and microarray based testing have enabled the testing of more than one disease in perhaps no time. With controlled synthesis, even magnetic nanoparticles have been synthesized and used for isolating and heavy metals resembling in properties with iron from the blood serum of living organisms. The evaluation of biochemical responses has been highly versatile and it has been so selective with the use of magnetic nanoparticles. This argues well for blood related disorders considering the involvement of iron protein hemoglobin. Such invasions have been collectively termed as diagnostic magnetic resonance as they use the optimization of magnetic coupling to the in vivo antigens of the body [62]. Much more sophisticated responses have been observed for detection via nanobiosensors using different ways of their incorporation in sensing mechanisms.

(2) Environmental Applications. This is a relatively broader area of application. This is so as environment undergoes so many rapid scale changes almost every second. The detection of pollutants, toxic intermediates, heavy metals from waste streams and the monitoring of weather conditions like the estimation of humidity and many other vital features are really highly detailed and comprehensive tasks. The sensors based on nanomaterials can be very versatile in terms of their detection and monitoring. The use of devices such as cantilever based electronic probes and the provisions which require very little amount of analyte are very good invaders of the technology. The nanomaterials based sensing tools can be used to find the particular kind of damaging extent of a material present or prevailing in the environment. In one such study, a Chinese hamster ovary cell line has been coupled with fluorescent reporter system and used to monitor various toxicants in highly diverse aqueous environments. Carcinogens and harmful intermediates leading to the disruption of proper hormonal systems in the living beings have been isolated through the use of highly sophisticated and specific compounds, particularly named as endocrinedisrupting compounds [63]. Similarly, in one such study, Purohit et al. have used biosensors to monitor the abiotic conditions that are essential for optimization of biological recovery applications like those of bioremediation [64]. In this way, the technique of bioremediation can be scaled up and used to optimize the environmental quality and decontaminate the hazardous contaminants. These applications when engineered with the use of nanomaterials can be far more useful and beneficial. Using the substrate specific detection mechanism, biosensors have been developed for detection of nitrates [65], inorganic phosphates [66, 67], and biological oxygen demand like parameters and have been proved to be environmentally restoring in their working mechanisms. These applications can be integrated and a single sensor can be developed by the use of nanomaterials which can sense the different contaminants equally well in only a single operation. In this manner, there are endless environmental parameters for the evaluation of which the nanobiosensors can be used and developed. These applications are highly energy saving, economical and time saving in nature.

(3) Miscellaneous Applications. Nanobiosensors can also be employed to optimize several other detections. In the industrial operations, feeding of nutrient media and substrate mixtures into the bioreactors for diverse applications can be regulated using these sensors. On an industrial scale, many commercial preparations and separations can be enhanced with these sensors. For instance, in the metallurgical operations requiring separation of impurities existing in a complexed form combined in the form of ores, nanobiosensors can be used to separate the impurities selectively by trying out different configurations of the sensing enzymes. Developing microbiological and biochemical assays coupled with bioengineering based innovations are really very handy applications of these sensing materials.

\section{Conclusion and Future Prospects}

Nanotechnology has really proved to be a very significant blessing in the development of biosensors. It has revolutionized the case of biological detection. The overall mechanisms have become quicker, smarter, less costly, and user friendly. The transduction mechanisms have been significantly improved with the use of nanomaterials and nanostructures like those of quantum dots, nanoparticles for enzyme immobilization, and hybrid nanostructures with multiple functionalities. Future argues very well for these dynamic, versatile, and quick recognition systems considering their multidimensional potential. These materials are right now being increasingly considered for the merging of chemical and biological sensors to make the overall process fast, easy to execute, and better in terms of performance $[68,69]$. The increasing advancement of miniaturization and nanomaterials research has stimulated the application of these materials for sensing several key pathways and regulatory events. With the current progress and exhaustive research pace of nanomaterial exploration, the sensing technology has become more and more versatile, robust, and dynamic. No doubt, biosensor development for a task is still very cumbersome and costly due to its technical complexities, but the incorporation of nanomaterials has proved to be a big boon for this technology, chiefly due to its friendly and result oriented experimental support.

\section{References}

[1] V. Dzyadevych, V. N. Arkhypova, A. P. Soldatkin et al., "Amperometric enzyme biosensors: past, present and future 
Biocapteurs enzymatiques à transduction ampérométrique: passé, présent, futur," Intermediate-Range Ballistic Missile, vol. 29, pp. 171-180, 2008.

[2] P. T. Kissinger, "Biosensors-a perspective," Biosensors and Bioelectronics, vol. 20, no. 12, pp. 2512-2516, 2005.

[3] C. Jianrong, M. Yuqing, H. Nongyue, W. Xiaohua, and L. Sijiao, "Nanotechnology and biosensors," Biotechnology Advances, vol. 22, no. 7, pp. 505-518, 2004.

[4] J. J. Davis, K. S. Coleman, B. R. Azamian, C. B. Bagshaw, and M. L. H. Green, "Chemical and biochemical sensing with modified single walled carbon nanotubes," Chemistry, vol. 9, no. 16, pp. 3732-3739, 2003.

[5] S. Sotiropoulou, V. Gavalas, V. Vamvakaki, and N. A. Chaniotakis, "Novel carbon materials in biosensor systems," Biosensors and Bioelectronics, vol. 18, no. 2-3, pp. 211-215, 2003.

[6] Y.-D. Zhao, W.-D. Zhang, H. Chen, Q.-M. Luo, and S. F. Y. Li, "Direct electrochemistry of horseradish peroxidase at carbon nanotube powder microelectrode," Sensors and Actuators B, vol. 87, no. 1, pp. 168-172, 2002.

[7] X. Luo, A. Morrin, A. J. Killard, and M. R. Smyth, "Application of nanoparticles in electrochemical sensors and biosensors," Electroanalysis, vol. 18, no. 4, pp. 319-326, 2006.

[8] E. Katz, I. Willner, and J. Wang, "Electroanalytical and bioelectroanalytical systems based on metal and semiconductor nanoparticles," Electroanalysis, vol. 16, no. 1-2, pp. 19-44, 2004.

[9] J. Wang, "Nanoparticle-based electrochemical DNA detection," Analytica Chimica Acta, vol. 500, no. 1-2, pp. 247-257, 2003.

[10] A. Merkoci, M. Aldavert, S. Marín et al., "New materials for electrochemical sensing. V: nanoparticles for DNA labeling," TrAC Trends in Analytical Chemistry, vol. 24, pp. 341-349, 2005.

[11] J. Wang, G. Liu, R. Polsky, and A. Merkoçi, "Electrochemical stripping detection of DNA hybridization based on cadmium sulfide nanoparticle tags," Electrochemistry Communications, vol. 4, no. 9, pp. 722-726, 2002.

[12] N. Zhu, A. Zhang, P. He, and Y. Fang, "Cadmium sulfide nanocluster-based electrochemical stripping detection of DNA hybridization," Analyst, vol. 128, no. 3, pp. 260-264, 2003.

[13] Y. Huang, W. Zhang, H. Xiao, and G. Li, "An electrochemical investigation of glucose oxidase at a CdS nanoparticles modified electrode," Biosensors and Bioelectronics, vol. 21, no. 5, pp. 817$821,2005$.

[14] Y. Cui, Q. Wei, H. Park, and C. M. Lieber, "Nanowire nanosensors for highly sensitive and selective detection of biological and chemical species," Science, vol. 293, no. 5533, pp. 1289-1292, 2001.

[15] E. Stern, J. F. Klemic, D. A. Routenberg et al., "Labelfree immunodetection with CMOS-compatible semiconducting nanowires," Nature, vol. 445, no. 7127, pp. 519-522, 2007.

[16] R. MacKenzie, V. Auzelyte, S. Olliges et al., "Nanowire development and characterization for applications in biosensing," Nanosystems Design and Technology, pp. 143-173, 2009.

[17] F. A. Rahim, Tunable Surface Assembly of Gold Nanorods for Biosensor Applications, Division of Bioengineering; Nanyang Technological University.

[18] A. V. Kabashin, P. Evans, S. Pastkovsky et al., "Plasmonic nanorod metamaterials for biosensing," Nature Materials, vol. 8, no. 11, pp. 867-871, 2009.

[19] S. Ramanathan, S. Patibandla, S. Bandyopadhyay, J. D. Edwards, and J. Anderson, "Fluorescence and infrared spectroscopy of electrochemically self assembled $\mathrm{ZnO}$ nanowires: evidence of the quantum confined Stark effect," Journal of Materials Science, vol. 17, no. 9, pp. 651-655, 2006.
[20] L. Chen, B. Gu, G. Zhu, Y. Wu, S. Liu, and C. Xu, "Electron transfer properties and electrocatalytic behavior of tyrosinase on ZnO nanorod," Journal of Electroanalytical Chemistry, vol. 617, no. 1, pp. 7-13, 2008.

[21] F. Qu, M. Yang, Y. Lu, G. Shen, and R. Yu, "Amperometric determination of bovine insulin based on synergic action of carbon nanotubes and cobalt hexacyanoferrate nanoparticles stabilized by EDTA," Analytical and Bioanalytical Chemistry, vol. 386, no. 2, pp. 228-234, 2006.

[22] P. Van Gerwen, W. Laureyn, W. Laureys et al., "Nanoscaled interdigitated electrode arrays for biochemical sensors," Sensors and Actuators B, vol. B49, no. 1-2, pp. 73-80, 1998.

[23] S. C. Pak, W. Penrose, and P. J. Hesketh, "An ultrathin platinum film sensor to measure biomolecular binding," Biosensors and Bioelectronics, vol. 16, no. 6, pp. 371-379, 2001.

[24] B. Bhushan, "Nanotribology and nanomechanics of MEMS/ NEMS and BioMEMS/BioNEMS materials and devices," Microelectronic Engineering, vol. 84, no. 3, pp. 387-412, 2007.

[25] S. Zeng, K.-T. Yong, I. Roy, X.-Q. Dinh, X. Yu, and F. Luan, "A review on functionalized gold nanoparticles for biosensing applications," Plasmonics, vol. 6, no. 3, pp. 491-506, 2011.

[26] K. L. Kelly, E. Coronado, L. L. Zhao, and G. C. Schatz, "The optical properties of metal nanoparticles: the influence of size, shape, and dielectric environment," Journal of Physical Chemistry B, vol. 107, no. 3, pp. 668-677, 2003.

[27] A. J. Haes and R. P. Van Duyne, "A nanoscale optical biosensor: sensitivity and selectivity of an approach based on the localized surface plasmon resonance spectroscopy of triangular silver nanoparticles," Journal of the American Chemical Society, vol. 124, no. 35, pp. 10596-10604, 2002.

[28] N. Nath and A. Chilkoti, "A colorimetric gold nanoparticle sensor to interrogate biomolecular interactions in real time on a surface," Analytical Chemistry, vol. 74, no. 3, pp. 504-509, 2002.

[29] R. Chakrabarti and A. M. Klibanov, "Nanocrystals modified with peptide nucleic acids (PNAs) for selective self-assembly and DNA detection," Journal of the American Chemical Society, vol. 125, no. 41, pp. 12531-12540, 2003.

[30] J. I. Reyes De Corcuera and R. P. Cavalieri, "Encyclopedia of Agricultural, Food and Biological Engineering".

[31] X. Su, F. T. Chew, and S. F. Y. Li, "Design and application of piezoelectric quartz crystal-based immunoassay," Analytical Sciences, vol. 16, no. 2, pp. 107-114, 2000.

[32] T. Liu, J. Tang, and L. Jiang, "The enhancement effect of gold nanoparticles as a surface modifier on DNA sensor sensitivity," Biochemical and Biophysical Research Communications, vol. 313, no. 1, pp. 3-7, 2004.

[33] J. Richardson, P. Hawkins, and R. Luxton, "The use of coated paramagnetic particles as a physical label in a magnetoimmunoassay," Biosensors and Bioelectronics, vol. 16, no. 9-12, pp. 989-993, 2001.

[34] Y. R. Chemla, H. L. Grossman, Y. Poon et al., "Ultrasensitive magnetic biosensor for homogeneous immunoassay," Proceedings of the National Academy of Sciences of the United States of America, vol. 97, no. 26, pp. 14268-14272, 2000.

[35] H. Cai, C. Xu, P. He, and Y. Fang, "Colloid Au-enhanced DNA immobilization for the electrochemical detection of sequencespecific DNA," Journal of Electroanalytical Chemistry, vol. 510, no. 1-2, pp. 78-85, 2001.

[36] A. L. Crumbliss, S. C. Perine, J. Stonehuerner, K. R. Tubergen, J. Zhao, and R. W. Henkens, "Colloidal gold as a biocompatible immobilization matrix suitable for the fabrication of enzyme 
electrodes by electrodeposition," Biotechnology and Bioengineering, vol. 40, no. 4, pp. 483-490, 1992.

[37] J. Zhao, J. P. O’Daly, R. W. Henkens, J. Stonehuerner, and A. L. Crumbliss, "A xanthine oxidase/colloidal gold enzyme electrode for amperometric biosensor applications," Biosensors and Bioelectronics, vol. 11, no. 5, pp. 493-502, 1996.

[38] X. Xu, S. Liu, and H. Ju, "A novel hydrogen peroxide sensor via the direct electrochemistry of horseradish peroxidase immobilized on colloidal gold modified screen-printed electrode," Sensors, vol. 3, no. 9, pp. 350-360, 2003.

[39] M. L. Curri, A. Agostiano, G. Leo, A. Mallardi, P. Cosma, and M. Della Monica, "Development of a novel enzyme/semiconductor nanoparticles system for biosensor application," Materials Science and Engineering C, vol. 22, no. 2, pp. 449-452, 2002.

[40] M. B. González-García, C. Fernández-Sánchez, and A. Costa-García, "Colloidal gold as an electrochemical label of streptavidin-biotin interaction," Biosensors and Bioelectronics, vol. 15, no. 5-6, pp. 315-321, 2000.

[41] J. J. Davis, K. S. Coleman, B. R. Azamian, C. B. Bagshaw, and M. L. H. Green, "Chemical and biochemical sensing with modified single walled carbon nanotubes," Chemistry, vol. 9, no. 16, pp. 3732-3739, 2003.

[42] S. Sotiropoulou, V. Gavalas, V. Vamvakaki, and N. A. Chaniotakis, "Novel carbon materials in biosensor systems," Biosensors and Bioelectronics, vol. 18, no. 2-3, pp. 211-215, 2002.

[43] B. R. Azamian, J. J. Davis, K. S. Coleman, C. B. Bagshaw, and M. L. H. Green, "Bioelectrochemical single-walled carbon nanotubes," Journal of the American Chemical Society, vol. 124, no. 43, pp. 12664-12665, 2002.

[44] A. Guiseppi-Elie, C. Lei, and R. H. Baughman, "Direct electron transfer of glucose oxidase on carbon nanotubes," Nanotechnology, vol. 13, no. 5, pp. 559-564, 2002.

[45] J. Wang, "Carbon-nanotube based electrochemical biosensors: a review," Electroanalysis, vol. 17, no. 1, pp. 7-14, 2005.

[46] Y. Cui and C. M. Lieber, "Functional nanoscale electronic devices assembled using silicon nanowire building blocks," Science, vol. 291, no. 5505, pp. 851-853, 2001.

[47] J. Wang, "Electrochemical nucleic acid biosensors," Analytica Chimica Acta, vol. 469, no. 1, pp. 63-71, 2002.

[48] B. M. Cullum, G. D. Griffin, G. H. Miller, and T. Vo-Dinh, "Intracellular measurements in mammary carcinoma cells using fiber-optic nanosensors," Analytical Biochemistry, vol. 277, no. 1, pp. 25-32, 2000.

[49] A. Umar, M. M. Rahman, A. Al-Hajry, and Y.-B. Hahn, "Highlysensitive cholesterol biosensor based on well-crystallized flower-shaped ZnO nanostructures," Talanta, vol. 78, no. 1, pp. 284-289, 2009.

[50] S. Yang, "Nanowires and nanobelts, materials, properties and devices," in Nanowires and Nanobelts of Functional Materials, Z. L. Wang, Ed., vol. 2, pp. 209-238, Kluwer Academic, New York, NY, USA, 2003.

[51] Y. Cui, Q. Wei, H. Park, and C. M. Lieber, "Nanowire nanosensors for highly sensitive and selective detection of biological and chemical species," Science, vol. 293, no. 5533, pp. 1289-1292, 2001.

[52] B. Huang, F. Yu, and R. N. Zare, "Surface plasmon resonance imaging using a high numerical aperture microscope objective," Analytical Chemistry, vol. 79, no. 7, pp. 2979-2983, 2007.

[53] E. Stern, J. F. Klemic, D. A. Routenberg et al., "Labelfree immunodetection with CMOS-compatible semiconducting nanowires," Nature, vol. 445, no. 7127, pp. 519-522, 2007.
[54] J. C. Pickup, F. Hussain, N. D. Evans, and N. Sachedina, "In vivo glucose monitoring: the clinical reality and the promise," Biosensors and Bioelectronics, vol. 20, no. 10, pp. 1897-1902, 2005.

[55] J. Bolinder, U. Ungerstedt, and P. Arner, "Microdialysis measurement of the absolute glucose concentration in subcutaneous adipose tissue allowing glucose monitoring in diabetic patients," Diabetologia, vol. 35, no. 12, pp. 1177-1180, 1992.

[56] T. G. Drummond, M. G. Hill, and J. K. Barton, "Electrochemical DNA sensors," Nature Biotechnology, vol. 21, pp. 1192-1199, 2003.

[57] L. G. Fägerstam, A. Frostell, R. Karlsson et al., "Detection of antigen-antibody interactions by surface plasmon resonance. Application to epitope mapping," Journal of Molecular Recognition, vol. 3, no. 5-6, pp. 208-214, 1990.

[58] M. Alterman, H. Sjobom, P. Safsten et al., "P1/P1/ modified HIV protease inhibitors as tools in two new sensitive surface plasmon resonance biosensor screening assays," European Journal of Pharmaceutical Sciences, vol. 13, no. 2, pp. 203-212, 2001.

[59] X. Gao, Y. Cui, R. M. Levenson, L. W. K. Chung, and S. Nie, "In vivo cancer targeting and imaging with semiconductor quantum dots," Nature Biotechnology, vol. 22, no. 8, pp. 969976, 2004.

[60] M. G. Harisinghani, J. Barentsz, P. F. Hahn et al., "Noninvasive detection of clinically occult lymph-node metastases in prostate cancer," The New England Journal of Medicine, vol. 348, no. 25, pp. 2491-2499, 2003.

[61] J. Grimm, J. M. Perez, L. Josephson, and R. Weissleder, "Novel nanosensors for rapid analysis of telomerase activity," Cancer Research, vol. 64, no. 2, pp. 639-643, 2004.

[62] J. B. Haun, T.-J. Yoon, H. Lee, and R. Weissleder, "Magnetic nanoparticle biosensors," Wiley Interdisciplinary Reviews, vol. 2, no. 3, pp. 291-304, 2010.

[63] E. J. Kim, Y. Lee, J. E. Lee, and M. B. Gu, "Application of recombinant fluorescent mammalian cells as a toxicity biosensor," Water Science and Technology, vol. 46, no. 3, pp. 51-56, 2002.

[64] H. J. Purohit, "Biosensors as molecular tools for use in bioremediation," Journal of Cleaner Production, vol. 11, no. 3, pp. 293301, 2003.

[65] L. H. Larsen, T. Kjær, and N. P. Revsbech, "A microscale $\mathrm{NO}_{3}$ - biosensor for environmental applications," Analytical Chemistry, vol. 69, no. 17, pp. 3527-3531, 1997.

[66] J. Kulys, I. J. Higgins, and J. V. Bannister, "Amperometric determination of phosphate ions by biosensor," Biosensors and Bioelectronics, vol. 7, no. 3, pp. 187-191, 1992.

[67] U. Wollenberger, F. Schubert, and F. W. Scheller, "Biosensor for sensitive phosphate detection," Sensors and Actuators B, vol. 7, no. 1-3, pp. 412-415, 1992.

[68] J. Ponmozhi, C. Frias, T. Marques, and O. Frazão, "Smart sensors/actuators for biomedical applications: review," Measurement, vol. 45, no. 7, pp. 1675-1688, 2012.

[69] T. Pradeep and A. Anshup, "Noble metal nanoparticles for water purification: a critical review," Thin Solid Films, vol. 517, no. 24, pp. 6441-6478, 2009. 

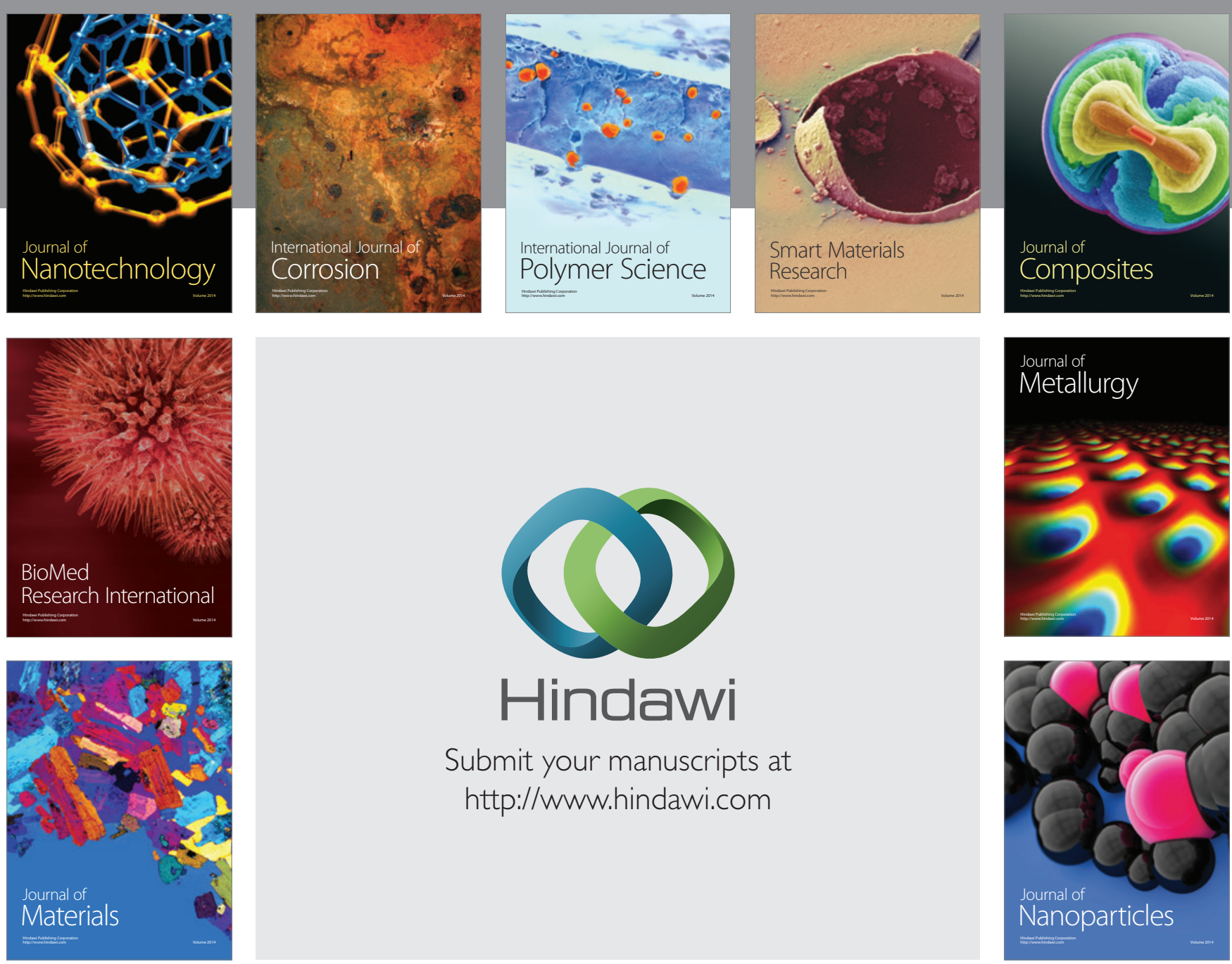

Submit your manuscripts at http://www.hindawi.com
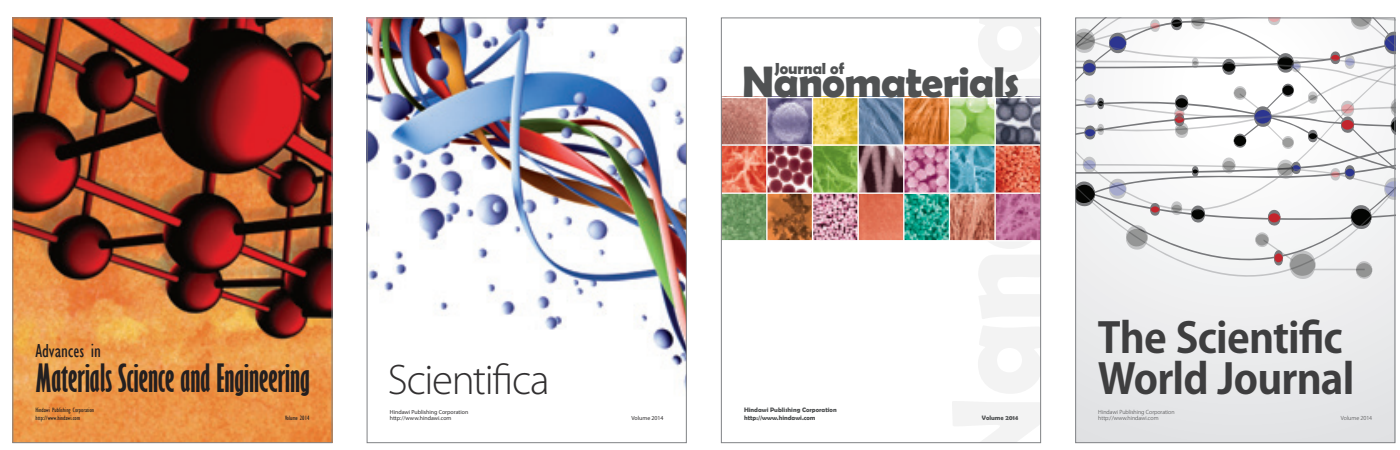

\section{The Scientific World Journal}
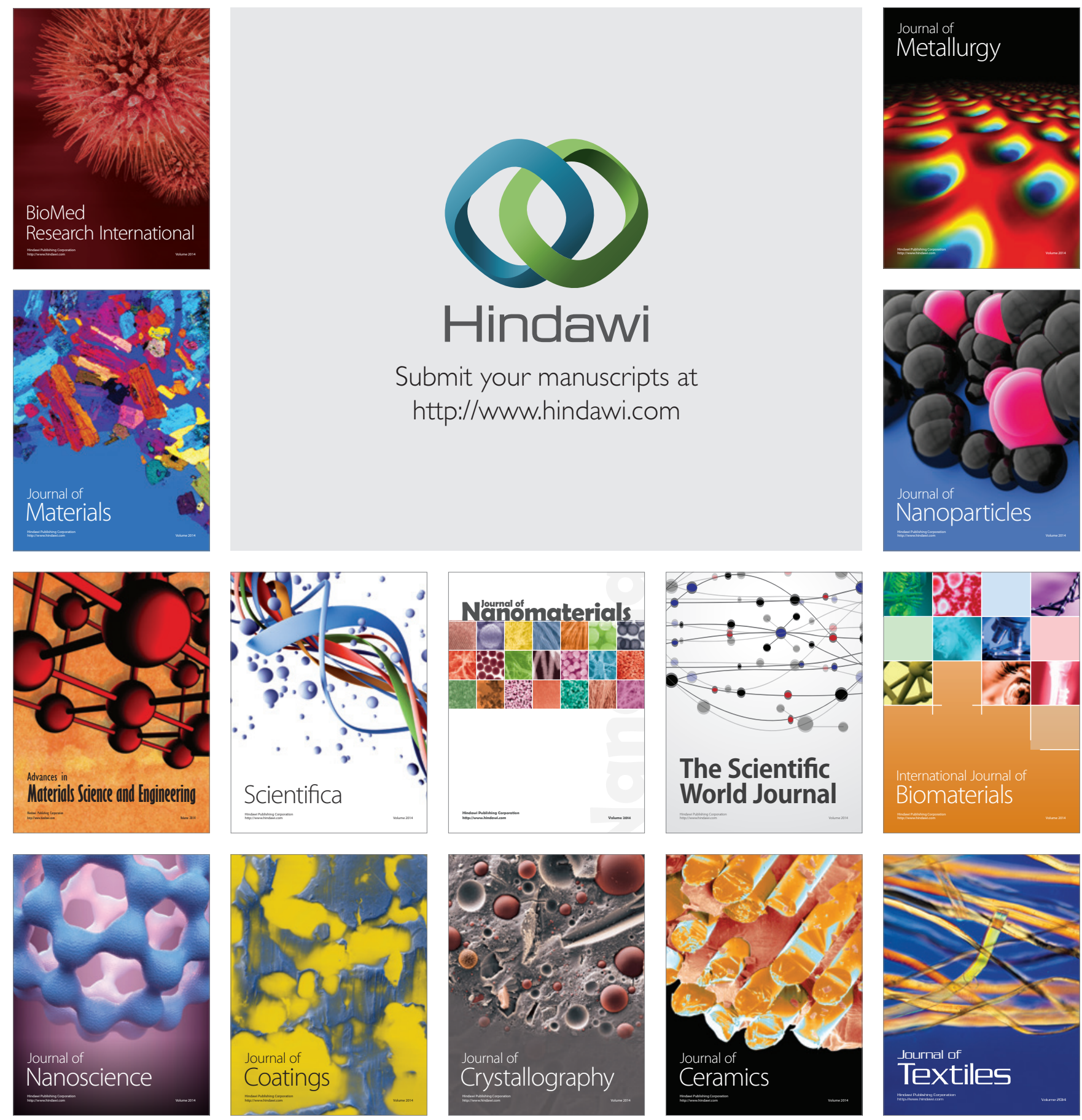